\title{
Dynamic Compression Behavior of ZK60 Magnesium Alloy under \\ Different Strain Rate
}

\author{
Zhu Shuiwen $^{1, a}$, Zhang wei ${ }^{1, b}$, Chen Guoping ${ }^{1, c^{*}}$ and Peng wei ${ }^{1, d}$ \\ ${ }^{1}$ College of Civil Eng \& Archi., Southwest University of Science and Technology, Mianyang,Sichuan, \\ P.R.China,621010 \\ aemail:40625727@qq.com, bemail:306361840@qq.com, \\ cemail:guopingchen2002@163.com, ${ }^{\mathrm{d}}$ email:weipengswust@163.com
}

Keywords: ZK60; Magnesium alloy; SHPB; Impact loading; Dynamic mechanical properties Abstract. To investigate dynamic compressive properties of ZK60 magnesium alloys under different strain rates, the Split Hopkinson Pressure Bar (SHPB) apparatus was used at ambient temperature. The mechanics response and the correlation rules of ZK60 magnesium alloy under different strain rate compression have been discussed in this paper. This research results show that ZK60 magnesium alloy has obvious yielding and strain strengthening under the high speed impact load, the maximum stress increases with the impact speed increasing, the dynamic yield strength is insensitive to the strain rate. In addition, with the increase of strain rate, distortion ability of the alloy has an extreme value.

\section{Introduction}

The increasing demand for better fuel economy, higher operating efficiency and related reduction of exhaust gases in automotive applications have prompted intensive research into lightweight structural materials. Magnesium alloy offers great potential for automobile construction and further technical applications in aeronautics, electronics and other fields due to its low density, high specific strength and high specific stiffness [1 2]. However, the limited ductility and poor formability of magnesium alloys at room temperature are often attributed to the difficulty in activating a sufficient number of independent slip and twinning systems.

The mechanical properties of magnesium alloy plate under quasi static loading condition have been investigated extensively [3 5]. However, experimental results of dynamic mechanical behavior of magnesium alloys at high strain rates are still far insufficient. This paper will fulfill the task focusing on the dynamic response of ZK60 magnesium under different strain rate using the Split Hopkinson Pressure Bar (SHPB) apparatus at ambient temperature.

\section{Experimental material and method}

The material was a commercially available ZK60 (mass fraction) hot rolled sheet, which was homogenized at 600 $\mathrm{K}$ for 1 hour, as shown in Table 1 . Cylinders $(\phi 18 \mathrm{~mm} \times 9 \mathrm{~mm})$ were cut from this sheet for compression testing with the cylinder.

\begin{tabular}{ccccccccc}
\hline $\mathrm{Si}$ & $\mathrm{Fe}$ & $\mathrm{Cu}$ & $\mathrm{Mn}$ & $\mathrm{Be}$ & $\mathrm{Ni}$ & $\mathrm{Zn}$ & $\mathrm{Zr}$ & $\mathrm{Mg}$ \\
\hline 0.0032 & 0.0020 & 0.00061 & 0.0039 & 0.00034 & $<0.0005$ & 6.37 & 0.53 & Bal. \\
\hline
\end{tabular}

Table 1 Chemical composition of ZK60 (mass fraction, \%) 
The high strain rate compression tests were carried out using Split Hopkinson Pressure Bar (SHPB). The experiment device and methods can be found in papers [6]. The SHPB system are is shown in Fig.1. The aim of this paper is to investigate the mechanical response of ZK60 magnesium alloy and related rules under different impact speed, which if from $15.3 \mathrm{~m} / \mathrm{s}$ to $25.6 \mathrm{~m} / \mathrm{s}$.

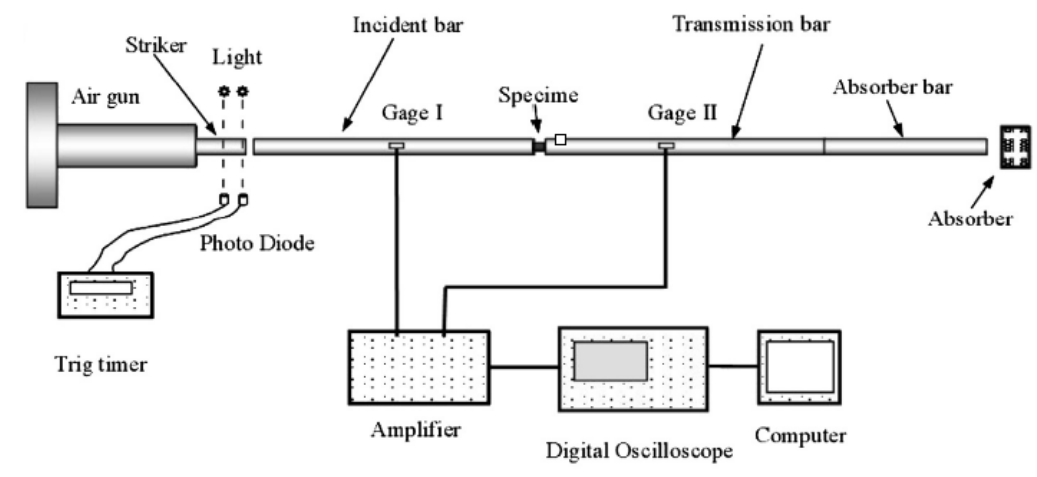

Fig.1. Schematic view of SHPB system

\section{Results and Discussions}

Stress-strain curve of ZK60 magnesium alloy under different impact speed: Based on the stress wave theory, the stress-strain curve under different impact speed is obtained by processing the original data, as shown Fig.2. The initial part of the curve in Fig.2 are all significant turning step, which shows that ZK60 magnesium alloy has obvious yield phenomenon under impact loading.

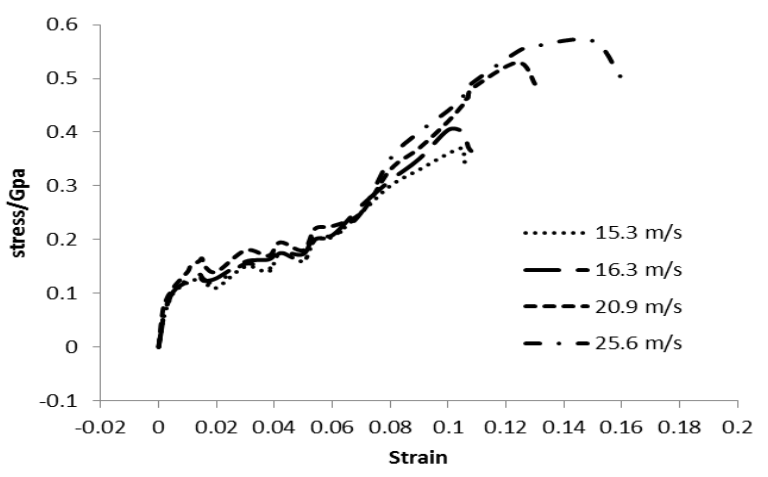

Fig.2. The stress-strain curve of ZK60 magnesium under different impact speed

The strain-hardening ability of the alloy under different impact speed was reflected in the curve, as shown in Fig.2. The stress-strain curve of high-impact-speed is located substantially above the lower-impact-speed, which shows that high impact speed of the sample is subjected to the greater stress in order to get the same strain. With the increase of impact speed, the degree of enhancement and the strain-hardening capacity of ZK60 magnesium alloy are increasing. Due to the rapid increase of stress pulse, the crystal twin is generated priority in the crystal. So the greater of the speed, the faster of the rise and the greater of the stress, this phenomenon is more obvious.

Dynamic yield strength and maximum stress under different impact speed: Relationships between the impact speed and the dynamic yield strength and the maximum stress values are presented in Fig.3. For general materials, the yield strength increase faster than the ultimate strength with the increase of impact speed, which makes the material brittle. 


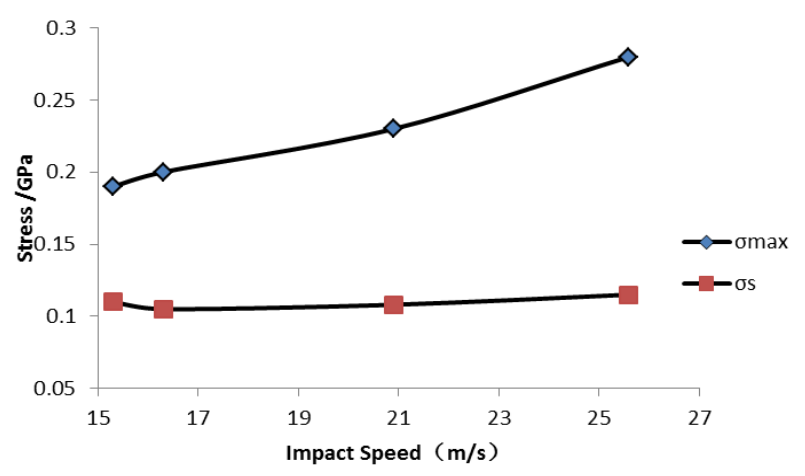

Fig.3. Dynamic yield strength and maximum stress under different impact speed

As can be seen from Fig.3, dynamic yield strength of ZK60 alloy has little change with the change of impact speed, and the maximum stress value increases as the impact speed increasing. But the increasing trend is gradually slowed down, resulting in the difference between the maximum stress and the dynamic yield strength increasing. Typically the yield strength and the ultimate strength of material increase with the strain rate increasing, and the increase of yield strength is much more significant than the ultimate strength, so that the material is easy to brittle fracture. The increasing differences of the yield strength and the maximum stress value indicate that the alloy material can absorb more energy after yield with the increase of impact speed.

Relationship between the deformation and the impact speed: The relationship between impact speed and the degree of deformation is obtained after measuring and calculating the amount of deformation of the sample under the impact load, as shown in Fig.4. The research results show that degree of deformation of the sample increases with the impact speed increasing, and the increasing amplitude is growing.

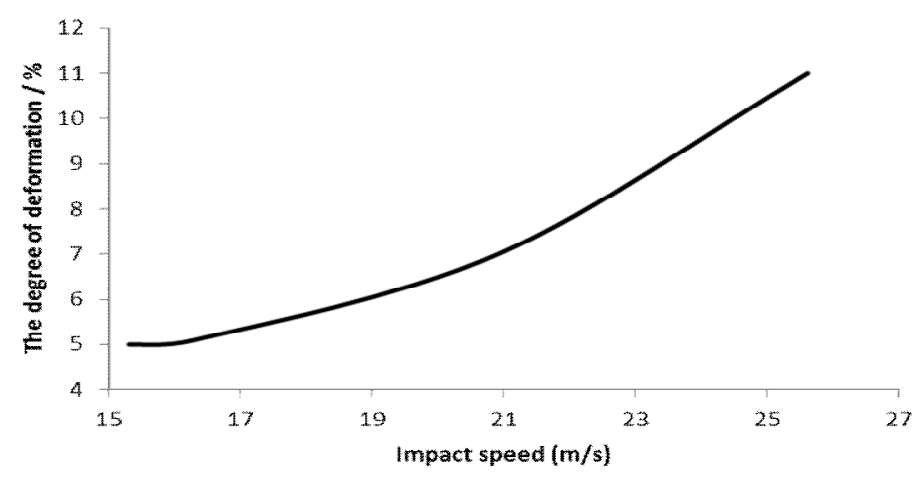

Fig.4. Deformation under different impact speed

Before rupturing, the deformability of ZK60 magnesium alloy is improved with the increasing of impact speed. Since the strain rate is a material deformation in response to the impact speed, it can be used as an indicator of the ability of the material deformation under impact load. The average strain rates exceed $100 \mathrm{~s}^{-1}$ in this experiment, which indicated the alloy is in the adiabatic condition during the impact [7]. The results showed that the average strain rate increase with the increase of impact speed, that is the deformation capacity of ZK60 magnesium alloy increasing with the impact speed increasing. However, the increase of the deformation ability is limited, because the experiments have shown that crack will appear when strain rate is more than $2000 \mathrm{~s}^{-1}$.

\section{Conclusion}

The aim of this study was to investigate dynamic compressive properties of ZK60 magnesium 
alloys under different strain rates. In this study,the Split Hopkinson Pressure Bar (SHPB) apparatus was used at ambient temperature. Based on the experimental study, the following conclusions are obtained.

(1) ZK60 magnesium alloy has obvious yield phenomenon and strain hardening phenomenon under impact loads, and its degree of enhancement is improved continuously with the increase of impact speed.

(2) The maximum stress of ZK60 magnesium alloy is increasing with the increasing of impact speed, but the dynamic yield strength is not sensitive to changes in strain rates.

(3) The increase of the deformation ability of ZK60 magnesium alloy is limited, the experiments show that crack will appear when strain rate is more than $2000 \mathrm{~s}^{-1}$.

(4) ZK60 magnesium alloys under dynamic compression yield continuously, and have the slight positive strain rate effect with increase of strain rates.

\section{References:}

[1] Li-he Chen, Hui-jie Zhao, Zheng Liu, Zhi-yong Shen, Yi-yong Nie,Die casting of magnesium alloy and its application in automobile industry,Foundry 10 (1999) 45-50.

[2] Bai-cheng Liu, Shou-mei Xiong,Blowup solutions and their blowup rates for parabolic equations with non-standard growth conditions, J. Automot. Saf. Energy 2 (1) (2011) 1-11.

[3] Yuan-xiang Shen, Xiu-rong Zhang, Ming-wen Luo,Bookmark and SharePrint this article Accelerating the Process Development and Information Construction of the Enterprise Using CAD/CAM, Journal of Sichuan Ordnance 29 (5) (2008) 114-116.

[4] Yu-juan Wu, Wen-jiang Ding, Li-ming Peng,Ignition-proof properties of a high-strength Mg-Gd-Ag-Zr alloy,etc.Materials China 30 (2) (2011) 1-9.

[5] Wei Ji, Ya-fu Fan, Jie Cheng, etc.,Effect of Temperature on the Dynamic Tensile Behavior and Fracture Mechanism of Mg-10Gd-2Y-0.5Zr Alloy, Journal of Materials Engineering 10 (2009) 41-44.

[6] Li Song, Shi-sheng Hu,Two-wave and three-wave method in SHPB data processing, J. Explosion Shock Waves 25 (4) (2005) 368-373.

[7] R.Z. Valiew, R.K. Islamgaliev, I.V. Alexandrov,Bulk nanostructured materials from severe plastic deformation, Prog. Mater. Sci. 45 (2000) 103-189. 